\title{
Behavior of a Wiener Integral along the Path of a Parameter $\mathrm{t}>0$
}

\author{
Kim Young Sik \\ Department of Mathematics, Research Institute of Natural Sciences, Industry -University Cooperation Foundation, Hanyang \\ University, 222 Wangsimni-ro, Seongdong-gu, Seoul 04763, Korea
}

\begin{abstract}
We investigate the behavior of a Wiener integral along the path of the scale factor as a function of a parameter for the Wiener integral about complex valued measurable function defined on the Wiener space which is frequently used in Quantum Mechanics.
\end{abstract}

Keywords-wiener space; wiener integral; feynman integral; fourier-stieltzes transform

\section{INTRODUCTION}

1. Motivation. In [3]-[5], R. H. Cameron and W. T. Martin proved some theorems on transformations and translations and used the expression of the change of scale for Wiener integrals (1944 - 1947).

In [2], R. H. Cameron wrote the paper about the translation pathology of Wiener space (1954).

In [1] M. D. Brue introduced the functional transform on Feynman integral (1972). In [10],G.W.Johnson and D. L. Skoug proved the scale-invariant measurability in Wiener space(1979)\}. In [7] - [8], R. H. Cameron and D. A. Storvick, proved relationships between the Wiener Integral and the analytic Feynman integral to prove a change of scale formula for Wiener Integrals (1987). In [11] - [12], Y. S. Kim proved Relationships between Wiener integrals and analytic Feynman Integrals and proved a change of scale formula for the Wiener integral for cylinder functions on the abstract Wiener space $(1998-2001)$.

In [13] - [16], Kim proved relationships among the Fourier transform and the Fourier Feynman transform and the convolution on the abstract Wiener space(2006 - 2016).

In [17], Kim investigated the interesting behavior of the scale factor for function space integrals and a Fourier Stieltjes transform on the Wiener space.

2. QUESTION. What is the mean of the transform and the change of scale for the Wiener integral?

3. TARGET. We re-interpret their papers about the change of scale and we investigate the behavior of a Wiener integral along the path $\mathbf{C}$ of the scale factor $\rho(t)$ as a function of a parameter $\mathbf{t}>\mathbf{0}$ about complex valued measurable function F: $\mathrm{C}_{0}[0, T] \rightarrow C$ defined on the Wiener space $C_{0}[0, T]$

$F(x)=\exp \left\{\int_{C_{0}[0, T]} \theta(t, x(t)) d t\right\}$ which is frequently used in Quantum Mechanics. From this query, we will find a very interesting behavior of the scale factor $\rho(t)$ for the
Wiener integral which varies very sensitively as a function of a parameter $\mathrm{t}>0$ !!

\section{DEFINITIONS AND PRELIMINARIES}

Let $\mathrm{C}_{0}[0, T]$ denote the space of real - valued continuous functions $\mathrm{x}$ on $[0, \mathrm{~T}]$ such that all real $x(0)=0$. Let $\mathrm{M}$ denote the class of all Wiener measurable subsets of $\mathrm{C}_{0}[0, T]$ and let $\mathrm{m}$ denote Wiener measure and $\left(\mathrm{C}_{0}[0, T], M, m\right)$ be Wiener measure space and we denote the Wiener integral of a functional $\mathrm{F}$ by $\cdot \int_{C_{0}[0, T]} F(x) d m(x)$

A subset $\mathrm{E}$ of $\mathrm{C}_{0}[0, T]$ is said to be scale-invariant measurable if $\rho(\mathrm{E}) \in M$ for each $\rho>0$ and a scale-invariant measurable set $\mathrm{N}$ is said to be scale-invariant null if $\mathrm{m}(\rho \mathrm{N})=$ 0 for each $\rho>0$. A property that holds except on a scaleinvariant null set is said to hold scale-invariant almost everywhere(s-a.e.). If two functionals $\mathrm{F}$ and $\mathrm{G}$ are equal s-a.e., we write $F \approx G$ ( For more details, see [9].

Throughout this paper, let $R^{n}$ denote the n-dimensional Euclidean space and let $\mathrm{C}$ and $C_{+} \quad$ and $C_{+} \sim$ denote the complex numbers, the complex numbers with positive real part, and the non-zero complex numbers with nonnegative real part, respectively.

Definition 2.1. Let F be a complex-valued measurable.

Function on $\mathrm{C}_{0}[0, T]$ such that the integral

$$
\mathrm{J}(\mathrm{F}: \mathrm{r})=\int_{C_{0}[0, T]} F\left(r^{-\frac{1}{2}} x\right) d m(x)
$$

exists for all real $r>0$. If there exists a function $J^{*}(F: z)$ analytic on $C_{+}$such that $J(F: r)=J^{*}(F: r)$ for all real $r>0$.

Then we define $J^{*}(F: z)$ to be the analytic Wiener integral of $\mathrm{F}$ over $C_{0}[0, T]$ with parameter $\mathrm{z}$ in $C_{+}$and for each $\mathrm{z}$ in $C_{+}$, we write $\int_{C_{0}[0, T]}^{a n w z} F(x) d m(x)=J^{*}(F: z)$

Let $\mathrm{q}$ be a non-zero real number and let $\mathrm{F}$ be a function on $C_{0}[0, T]$ whose analytic Wiener integral exists for each $\mathrm{z}$ in $C_{+}$.. If the following limit exists, then we call it the analytic Feynman integral of $\mathrm{F}$ over $C_{0}[0, T]$ with parameter q, and we write 


$$
\int_{C_{0}[0, T]}^{a f} F(x) d m(x)=\lim _{\mathrm{z} \rightarrow-\mathrm{iq}} \int_{C_{0}[0, T]}^{a n w z} F(x) d m(x)
$$

where z approaches - iq through $C_{+}$and $i^{2}=-1$.

Now we introduce the following well-known Wiener Integration Formula.

Theorem2.2. Let $C_{0}[0, T]$ be a Wiener space and let

$$
0 \leq t_{1} \leq t_{2} \leq \cdots \leq t_{n} \leq T
$$

Then $\int_{C_{0}[0, T]} f\left(x\left(t_{1}\right), x\left(t_{2}\right), \cdots, x\left(t_{n}\right)\right) d m(x)$

$$
=\left(\prod_{j=1}^{n} 2 \pi\left(t_{j}-t_{j-1}\right)\right)^{-1 / 2}
$$

$$
\cdot \int_{R^{n}} f(\vec{u}) \exp \left\{-\frac{1}{2} \sum_{j=1}^{n} \frac{\left(u_{j}-u_{j-1}\right)^{2}}{t_{j}-t_{j-1}}\right\} d \vec{u}
$$

where $\mathrm{f}: \mathrm{R}^{\wedge} \mathrm{n} \rightarrow \mathrm{C}$ is a Lebesgue measurable function and

$$
\vec{u}=\left(u_{1}, u_{2}, \cdots, u_{n}\right), d \vec{u}=d u_{1} d u_{2} \cdots d u_{n} .
$$

In the next section, we will use several times the following well-known integration formula:

$$
\int_{R^{n}} \exp \left\{-a u^{2}+i b u\right\} d \vec{u}=\sqrt{\frac{\pi}{a}} \exp \left\{-\frac{b^{2}}{4 a}\right\}
$$

where $a$ is a complex number with the positive real part $(\operatorname{Re}(a)$ $>0$ ) and $\mathrm{b}$ is a real number and $i^{2}=-1$.

\section{BeHAVIOR OF A SCALE FActor For the Wiener INTEGRAL.}

Target of this paper : We re-interpret their papers about the change of scale and investigate the behavior of the scale factor about functions :

$$
F(x)=\exp \left\{\int_{C_{0}[0, T]} \theta(t, x(t)) d t\right\}
$$

which are frequently used in Quantum Mechanics. From this query, we will find a very interesting behavior of the scale factor and the change of scale factor for the Wiener integral!!

Definition 3.1 Let $\theta:[0, T] \times R \rightarrow C$ be defined by (3.2)

$$
\theta(t, u)=\int_{R} \exp \{i u v\} d \sigma_{t}(v)
$$

which is a Fourier-Stieltzes transform of a complex Borel measure $\sigma_{t} \in M(R)$, where $\mathrm{M}(\mathrm{R})$ is a set of complex Borel measures defined on R.

Notation Let

$$
\Delta_{n} \equiv\left\{\left(t_{1}, t_{2}, \cdots, t_{n}\right) \mid 0 \leq t_{1} \leq t_{2} \leq \cdots \leq t_{n}=T\right\}, \quad t_{0}=0
$$

To expand the main result of this paper and to apply the Wiener integration formula and to prove the existence of the Wiener integral of $\mathrm{F}(\mathrm{x})$ in (3.1), we need to express $\mathrm{F}(\mathrm{x})$ as the function of the form: $f\left(x\left(t_{1}\right), x\left(t_{2}\right), \ldots, x\left(t_{n}\right)\right)$.

Lemma3.2. Let F: $C_{0}[0, T] \rightarrow C$ be defined by (3.1) and (3.2). Then we have that

$$
\mathrm{F}(\mathrm{x})=\sum_{n=0}^{\infty} \int_{\Delta_{n}(T) \times R^{n}} \exp \left\{i \sum_{j=1}^{n} v_{j} x\left(t_{j}\right) \quad\right\} d \mu_{n}(\vec{t}, \vec{v})
$$

where $\mu_{n}$ is a countably additive Borel measure defined on $\Delta_{n}(T) \times R^{n}$ for each $\mathrm{n}=1,2, \ldots \mathrm{n}$.

Proof. Using the series expansion of the exponential function,

$$
\begin{aligned}
& F(x)=\exp \left\{\int_{C_{0}[0, T]} \theta(t, x(t)) d t\right\} \\
& =\sum_{n=0}^{\infty} \frac{1}{n !}\left[\int_{0}^{T} \theta(t, x(t)) d t\right]^{n} \\
& =\sum_{n=0}^{\infty} \frac{1}{n !}\left[n ! \int_{\Delta_{n}(T)} \prod_{j=1}^{n} \theta\left(t_{j}, x\left(t_{j}\right)\right) d \vec{t}\right]^{n} \\
& =\sum_{n=0^{\infty}}^{\infty} \int_{\Delta_{n}(T)} \theta\left(t_{1}, x\left(t_{1}\right)\right) \cdots \theta\left(t_{n}, x\left(t_{n}\right)\right) d t_{1} \cdots d t_{n} \\
& =\sum_{n=0} \int_{\Delta_{n}(T)}\left[\int_{R} \exp \left\{i v_{1} x\left(t_{1}\right)\right\} d \sigma_{t_{1}}\left(v_{1}\right)\right] \\
& \quad \cdots \int_{\Delta_{n}(T)}\left[\int_{R} \exp \left\{i v_{n} x\left(t_{n}\right)\right\} d \sigma_{t_{n}}\left(v_{n}\right)\right] d t_{1} \cdots d t_{n} \\
& =\sum_{n=0}^{\infty} \int_{\Delta_{n}(T) \times R^{n}} \exp \left\{i \sum_{j=1}^{n} v_{j} x\left(t_{j}\right)\right\} d \mu_{n}(\vec{t}, \vec{v})
\end{aligned}
$$

where $d \mu_{n}(t, \vec{v})=\prod_{j=1}^{n}\left[d \sigma_{t_{j}}\left(v_{j}\right)\right] d\left(t_{j}\right)$. And $\sigma_{t_{j}}$ is a complex Borel measure defined on $\mathrm{R}$ for each $\mathrm{j}=1,2, \ldots, \mathrm{n}$. Note that $|F(x)| \leq \sum_{n=1}^{\infty}\left\|\mu_{n}\right\|<\infty \cdots$.

Theorem3.3. For a function F: $C_{0}[0, T] \rightarrow C$ be defined by (3.1) and (3.2) and for real $\rho>0$,

$$
\begin{aligned}
& \int_{C_{0}[0, T]} F(\rho x) d m(x) \\
= & \sum_{n=0}^{\infty} \int_{\Delta_{n}(T) \times R^{n}} \exp \left\{-\frac{\rho^{2}}{2} \sum_{j=1}^{n}\left(t_{j}-t_{j-1}\right) \sum_{k=j}^{n} v_{k}^{2}\right\} d \mu_{n}(\vec{t}, \vec{v})
\end{aligned}
$$


where $\mu_{n}$ a countably additive complex Borel measure defined on $\Delta_{n}(T) \times R^{n} \quad$ for each $\mathrm{n}=1,2, \ldots \quad$ and $d \mu_{n}(t, \vec{v})=$ $\prod_{j=1}^{n}\left[d \sigma_{t_{j}}\left(v_{j}\right)\right] d\left(t_{j}\right)$

Proof. By the Wiener integration formula, we have that for real $r>0$.

$$
\begin{aligned}
& \int_{C_{0}[o, T[} F\left(r^{-\frac{1}{2} \cdot x}\right) d m(x) \\
& =\int_{C_{0}[0, T]}\left[\sum_{n=0}^{\infty}\left[\prod_{j=1}^{n}\left[\int_{R} \exp \left\{i r^{-\frac{1}{2}} x\left(t_{j}\right) v_{j}\right\} d \sigma_{t_{j}}\left(v_{j}\right)\right] d \vec{t}\right]\right] d m(x) \\
& =\prod_{j=1}^{n}\left(\frac{r}{2 \pi\left(t_{j}-t_{j-1}\right)}\right)^{\frac{1}{2}} \int_{R^{n}}\left[\sum_{n=0}^{\infty} \int_{\Delta_{n}(T)}\right. \\
& {\left[\prod_{j=1}^{n} \int_{R} \exp \left\{\operatorname{ir}^{-\frac{1}{2}} u_{j} v_{j}\right\} d \sigma_{t_{j}}\left(v_{j}\right)\right] d \vec{t}} \\
& \cdot \exp \left\{-\frac{r}{2} \sum_{j=1}^{n} \frac{\left[u_{j}-u_{j-1}\right]^{2}}{t_{j}-t_{j-1}}\right\} d \vec{u} \\
& =\prod_{j=1}^{n}\left(\frac{r}{2 \pi\left(t_{j}-t_{j-1}\right)}\right)^{\frac{1}{2}} \int_{R^{n}} \exp \left\{-\frac{r}{2} \sum_{j=1}^{n} \frac{\left[u_{j}-u_{j-1}\right]^{2}}{t_{j}-t_{j-1}}\right\} \\
& \cdot\left[\sum_{n=0}^{\infty} \int_{\Delta_{n}(T) \times R^{n}} \exp \left\{i \sum_{j=1}^{n} u_{j} v_{j}\right\}\left\lceil\prod_{j=1}^{n} d \sigma_{t_{j}}\left(v_{j}\right) d t_{j}\right\rceil d \vec{u}\right. \\
& =\prod_{j=1}^{n}\left(\frac{r}{2 \pi\left(t_{j}-t_{j-1}\right)}\right)^{\frac{1}{2}} \int_{R^{n}} \exp \left\{-\frac{r}{2} \sum_{j=1}^{n} \frac{\left[u_{j}-u_{j-1}\right]^{2}}{t_{j}-t_{j-1}}\right\} \\
& \cdot\left[\sum_{n=0}^{\infty} \int_{\Delta_{n}(T) \times R^{n}} \exp \left\{i \sum_{j=1}^{n} u_{j} v_{j}\right\} d \mu_{n}(\vec{t}, \vec{v})\right] d \vec{u} \\
& =\left[\sum_{n=0}^{\infty} \int_{\Delta_{n}(T) \times R^{n}} \prod_{j=1}^{n}\left(\frac{r}{2 \pi\left(t_{j}-t_{j-1}\right)}\right)^{\frac{1}{2}}\right. \\
& \cdot \int_{R^{n}} \exp \left\{-\frac{r}{2} \sum_{j=1}^{n} \frac{\left[u_{j}-u_{j-1}\right]^{2}}{t_{j}-t_{j-1}}\right\} \cdot \exp \left\{i \sum_{j=1}^{n} u_{j} v_{j}\right\} d \mu_{n}(\vec{t}, \vec{v}) \\
& =\sum_{n=0}^{\infty} \int_{\Delta_{n}(T) \times R^{n}} \exp \left\{-\frac{1}{2 r} \sum_{j=1}^{n}\left(t_{j}-t_{j-1}\right) \sum_{k=j}^{n} v_{k}^{2}\right\} d \mu_{n}(\vec{t}, \vec{v})
\end{aligned}
$$

where the last equality in (3.6) can be proved by the mathematical induction. If we let $r=\rho^{-2}$, then for real $\rho>0$,

$$
\begin{aligned}
& \int_{C_{0}[0, T]} F(\rho x) d m(x) \\
= & \sum_{n=0}^{\infty} \int_{\Delta_{n}(T) \times R^{n}} \exp \left\{-\frac{\rho^{2}}{2} \sum_{j=1}^{n}\left(t_{j}-t_{j-1}\right) \sum_{k=j}^{n} v_{k}^{2}\right\} d \mu_{n}(\vec{t}, \vec{v})
\end{aligned}
$$

By the above result, we can investigate a very interesting behavior of the scale factor for the Wiener integral :

\section{Behavior of a Wiener integral upon the scale factor}

Definition 3.4. We define the scale factor for the Wiener integral by the varying real number $\rho>0$, such that

$$
G(\rho)=\left|\int_{C_{0}[o, T[} F(\rho x) d m(x)\right|
$$

where $\mathrm{G}: \mathrm{R} \rightarrow \mathrm{C}$ is a complex valued function defined on $\mathrm{R}$.

To investigate behavior of the scale factor for the Wiener integral by the graphical meaning, we need the following definition.

Definition3.5. We define the spectrum for the scale factor

$\rho>0$ of the Wiener integral by

$$
\begin{aligned}
& \operatorname{Spec}_{\rho}\left\{\int_{C_{0}[o, T[} F(x) d m(x)\right\} \\
& =\left\{\left(\rho,\left|\int_{C_{0}[o, T[} F(\rho x) d m(x)\right|\right) \mid \rho>0\right\}
\end{aligned}
$$

\section{BEHAVIOR OF A SCALE FACTOR FOR THE WIENER} INTEGRAL AS A FUNCTION OF A PARAMETER T $>0$.

In this section, we discuss the main target of this paper. We wish to investigate the behavior of the Wiener integral upon the scale factor $\rho>0$ as a parameter $t>0$.

Definition 4.1. Define a function $G:(0, \infty) \rightarrow$ R. by

$$
\begin{aligned}
& G(t)=\int_{C_{0}[o, T[} F(\rho(t) x) d m(x) \\
& =\sum_{n=0}^{\infty} \int_{\Delta_{n}(T) \times R^{n}} \exp \left\{-\frac{\rho(t)^{2}}{2} \sum_{j=1}^{n}\left(t_{j}-t_{j-1}\right) \sum_{k=j}^{n} v_{k}^{2}\right\} d \mu_{n}(\vec{t}, \vec{v})
\end{aligned}
$$

To investigate the behavior of the scale factor for the Wiener integral more precisely, we take some paths $\rho(t)$.which varies as a parameter $t>0$. 
(1). For a parameter $t>0$,

$$
\begin{aligned}
& \int_{C_{0}[o, T[} F(\rho(t) x) d m(x) \\
& =\sum_{n=0}^{\infty} \int_{\Delta_{n}(T) \times R^{n}} \exp \left\{-\frac{\rho(t)^{2}}{2} \sum_{j=1}^{n}\left(t_{j}-t_{j-1}\right) \sum_{k=j}^{n} v_{k}^{2}\right\} d \mu_{n}(\vec{t}, \vec{v})
\end{aligned}
$$

(2) Whenever the scale factor $\rho>0$ is increasing along the path $\rho(\mathrm{t})=t^{2}(t>0)$ varying as a function of a parameter $t>0$, the Wiener integral is decreasing :

$$
\begin{aligned}
& \int_{C_{0}[o, T[} F\left(t^{2} x\right) d m(x) \\
= & \sum_{n=0}^{\infty} \int_{\Delta_{n}(T) \times R^{n}} \exp \left\{-\frac{t^{4}}{2} \sum_{j=1}^{n}\left(t_{j}-t_{j-1}\right) \sum_{k=j}^{n} v_{k}^{2}\right\} d \mu_{n}(\vec{t}, \vec{v})
\end{aligned}
$$

(3) Whenever the scale factor $\rho>0$ is increasing along the path $\rho(\mathrm{t})=t^{4}(t>0)$ varying as a function of a parameter $t>0$, the Wiener integral is decreasing :

$$
\begin{aligned}
& \int_{C_{0}[o, T[} F\left(t^{4} x\right) d m(x) \\
= & \sum_{n=0}^{\infty} \int_{\Delta_{n}(T) \times R^{n}} \exp \left\{-\frac{t^{8}}{2} \sum_{j=1}^{n}\left(t_{j}-t_{j-1}\right) \sum_{k=j}^{n} v_{k}^{2}\right\} d \mu_{n}(\vec{t}, \vec{v})
\end{aligned}
$$

It decreases more rapidly than the curve of a scale factor $\rho(\mathrm{t})=t^{2}(t>0)$

(4) Whenever the parameter $t>1$ is increases up to $t=\infty$ along the path of a scale factor $\rho(\mathrm{t})=t^{4}(t>0)$, the Wiener integral is decreasing up to 0 :

$$
\lim _{t \rightarrow \infty} \int_{C_{0}[o, T[} F(\rho(t) x) d m(x)=0
$$

(5). Therefore the Wiener integral along the path of a scale factor $\rho(\mathrm{t})=t^{4}(t>1)$ is a decreasing function of a parameter $t>1$ and

$$
0 \leq \int_{C_{0}[o, T[} F(\rho(t) x) d m(x) \leq \sum_{n=0}^{\infty}\left\|\mu_{n}\right\|
$$

(6) Whenever the scale factor $\rho>0$ moves along the path $\rho(\mathrm{t})=\frac{1}{t^{2}}(t>0)$ varying as a function of a parameter $\mathrm{t}>0$, the Wiener integral is increasing :

$$
\int_{C_{0}[o, T[} F\left(\frac{1}{t^{2}} x\right) d m(x)
$$

$$
=\sum_{n=0}^{\infty} \int_{\Delta_{n}(T) \times R^{n}} \exp \left\{-\frac{1}{2 t^{4}} \sum_{j=1}^{n}\left(t_{j}-t_{j-1}\right) \sum_{k=j}^{n} v_{k}^{2}\right\} d \mu_{n}(\vec{t}, \vec{v})
$$

(7) Whenever the scale factor $\rho>0$ varies along the path $\rho(\mathrm{t})=\frac{1}{t^{2}}(t>0)$ as a function of a parameter $\mathrm{t}>0$, the Wiener integral $\mathrm{g}(\rho(\mathrm{t}))=\int_{C_{0}[o, T[} F(\rho(t) x) d m(x)$ is an increasing function of a parameter $\mathrm{t}>0$ :

(a) $0<t_{1}<t_{2}=>\rho\left(t_{1}\right)>\rho\left(t_{2}\right)=>e^{-\rho\left(t_{1}\right)^{2}}<e^{-\rho\left(t_{2}\right)^{2}}<1$

(b)

$$
\begin{aligned}
& \sum_{n=0}^{\infty} \int_{\Delta_{n}(T) \times R^{n}} \exp \left\{-\frac{\rho\left(t_{1}\right)^{2}}{2} \sum_{j=1}^{n}\left(t_{j}-t_{j-1}\right) \sum_{k=j}^{n} v_{k}^{2}\right\} d \mu_{n}(\vec{t}, \vec{v}) \\
& <\sum_{n=0}^{\infty} \int_{\Delta_{n}(T) \times R^{n}} \exp \left\{-\frac{\rho\left(t_{2}\right)^{2}}{2} \sum_{j=1}^{n}\left(t_{j}-t_{j-1}\right) \sum_{k=j}^{n} v_{k}^{2}\right\} d \mu_{n}(\vec{t}, \vec{v}) \\
& \text { (c) } \int_{C_{0}[o, T[} F\left(\rho\left(t_{1}\right) x\right) d m(x)<\int_{C_{0}[o, T[} F\left(\rho\left(t_{2}\right) x\right) d m(x) .
\end{aligned}
$$

(8) Whenever the scale factor $\rho>0$ varies along the path $\rho(\mathrm{t})=\frac{1}{t^{2}}(t>0)$ as a function of a parameter $\mathrm{t}>0$, the Wiener integral $\mathrm{g}(\rho(\mathrm{t}))=\int_{C_{0}[o, T[} F(\rho(t) x) d m(x)$ is an increasing function of a parameter $\mathrm{t}>0$ :

(9). Now, we compare the relationship between the behavior of the Wiener integral which varies along the path $C_{1}: \rho(\mathrm{t})=t^{2}(t>0)$ and the behavior of the Wiener integral which varies along the path $C_{2}: \rho(\mathrm{t})=t^{4}(t>0)$ :

$$
\begin{aligned}
& C_{1}: \int_{C_{0}[o, T[} F(\rho(t) x) d m(x) \\
& =\sum_{n=0}^{\infty} \int_{\Delta_{n}(T) \times R^{n}} \exp \left\{-\frac{t^{4}}{2} \sum_{j=1}^{n}\left(t_{j}-t_{j-1}\right) \sum_{k=j}^{n} v_{k}^{2}\right\} d \mu_{n}(\vec{t}, \vec{v}) .
\end{aligned}
$$

$$
C_{2}: \int_{C_{0}[o, T[} F(\rho(t) x) d m(x)
$$

$=\sum_{n=0}^{\infty} \int_{\Delta_{n}(T) \times R^{n}} \exp \left\{-\frac{t^{8}}{2} \sum_{j=1}^{n}\left(t_{j}-t_{j-1}\right) \sum_{k=j}^{n} v_{k}^{2}\right\} d \mu_{n}(\vec{t}, \vec{v})$

The Wiener integral along the path $C_{2}$ moves more rapidly than the Wiener integral along the path $C_{1}$ for a parameter $\mathrm{t}>$ 0 .

Example2. Define a function $\mathrm{F}: C_{0}[0, T] \rightarrow C$ by

$$
F(x)=\exp \left\{-\sum_{j=1}^{n}\left(\int_{0}^{T} \alpha_{j} d x\right)^{2}\right\}
$$


where $\left\{\alpha_{1}, \alpha_{2}, \cdots, \alpha_{n}\right\}$ is an orthonormal set of elements in $L_{2}[0, T]$.

Then $F(x)$ is Wiener integrable and

$$
\begin{aligned}
& \int_{C_{0}[o, T[} F(x) d m(x) \\
& =\left(\frac{1}{2 \pi}\right)^{\frac{n}{2}} \int_{R^{n}} \exp \left\{-\sum_{j=1}^{n} u_{j}^{2}-\frac{1}{2} \sum_{j=1}^{n} u_{j}^{2}\right\} d \vec{u} \\
& =\left(\frac{1}{2 \pi}\right)^{\frac{n}{2}} \cdot\left(\frac{2 \pi}{3}\right)^{\frac{n}{2}} \\
& =(\sqrt{3})^{-n} .
\end{aligned}
$$

For all real $r>0$, the Wiener integral is

$$
\begin{aligned}
& \int_{C_{0}[o, T[} F\left(r^{-\frac{1}{2}} x\right) d m(x) \\
& =\left(\frac{1}{2 \pi}\right)^{\frac{n}{2}} \int_{R^{n}} \exp \left\{-\frac{1}{r} \sum_{j=1}^{n} u_{j}^{2}-\frac{1}{2} \sum_{j=1}^{n} u_{j}^{2}\right\} d \vec{u} \\
& =\left(\frac{1}{2 \pi}\right)^{\frac{n}{2}} \int_{R^{n}} \exp \left\{-\frac{r+2}{2 r} \sum_{j=1}^{n} u_{j}^{2}\right\} d \vec{u} \\
& =\left(\frac{1}{2 \pi}\right)^{\frac{n}{2}} \cdot\left(\frac{2 \pi r}{r+2}\right)^{\frac{n}{2}} \\
& =\left(\frac{r}{2+r}\right)^{\frac{n}{2}} .
\end{aligned}
$$

(a). Behavior of a scale factor $\rho>0$ for the Wiener integral

(1). Define a function $G: R \rightarrow R$ by

$$
G(\rho)=\left|\int_{C_{0}[o, T[} F(\rho x) d m(x)\right|
$$

Where $\rho>0$ is a scale factor for the Wiener integral..

(2). $G(\rho)=\left(\frac{1}{1+2 \rho^{2}}\right)^{\frac{n}{2}}$, for $\rho>0$

(3). G( $\rho)$ is a decreasing function of a scale factor $\rho>0$.

(4). Whenever the scale factor $\rho>0$ is increasing, the Wiener integral decreases very rapidly.

(5). As the inflection point of the function $G(\rho)=$ $\left(\frac{1}{1+2 \rho^{2}}\right)^{\frac{n}{2}}(\rho>0)$ is $\rho=\frac{1}{\sqrt[2]{6}}$, the Wiener integral decreases very rapidly around the $\epsilon$ - neighborhood of the scale factor $\rho=\frac{1}{\sqrt[2]{6}}$

(6). The spectrum of the scale factor $\rho>0$ about the Wiener integral is a decreasing function of a scale factor $\rho>0$.

(b). Behavior of the scale factor $\rho(t)$ of a parameter $t>0$ which varies very vividly according to the parameter $t>0$.
(1). Whenever the scale factor $\rho>0$ is increasing along the path .

path $C_{1}: \rho(\mathrm{t})=t^{2}(t>0)$ varying as a parameter $t>0$, the Wiener integral is decreasing :

$$
\int_{C_{0}[o, T[} F\left(t^{2} x\right) d m(x)=\left(\frac{1}{1+2 t^{4}}\right)^{\frac{n}{2}}(t>0)
$$

And

$$
\lim _{t \rightarrow 0} \int_{C_{0}[o, T[} F\left(t^{2} x\right) d m(x)=1
$$

(2) Whenever the scale factor $\rho>0$ is increasing along the path .

$C_{2}: \rho(\mathrm{t})=t^{4}(t>0)$, the Wiener integral is decreasing :

$$
\int_{C_{0}[o, T[} F\left(t^{2} x\right) d m(x)=\left(\frac{1}{1+2 t^{8}}\right)^{\frac{n}{2}}(t>0)
$$

and

$$
\lim _{t \rightarrow 0} \int_{C_{0}[o, T[} F\left(t^{2} x\right) d m(x)=1
$$

It decreases more rapidly than the case of the path $C_{1}$ : $\rho(\mathrm{t})=t^{2}(t>0)$.

(3). Whenever the scale factor $\rho>0$ is decreasing along the path.

$C_{3}: \rho(\mathrm{t})=\frac{1}{t^{2}}(t>1)$, the Wiener integral is increasing :

$$
g(t)=\int_{C_{0}[o, T[} F\left(t^{2} x\right) d m(x)=\left(\frac{t^{4}}{t^{4}+2}\right)^{\frac{n}{2}}(t>0)
$$

and

$$
\lim _{t \rightarrow \infty} \int_{C_{0}[o, T[} F\left(\frac{1}{t^{2}} x\right) d m(x)=1
$$

It decreases very slowly, whenever a parameter $t>0$ is increasing.

(4). ). Whenever the scale factor $\rho>0$ is decreasing along the path $C_{4}: \rho(\mathrm{t})=\frac{1}{t^{4}}(t>1)$, the Wiener integral is increasing:

$$
h(t)=\int_{C_{0}[o, T[} F\left(\frac{1}{t^{4}} x\right) d m(x)=\left(\frac{t^{8}}{t^{8}+2}\right)^{\frac{n}{2}}(t>0)
$$

and

$$
\lim _{t \rightarrow \infty} \int_{C_{0}[o, T[} F\left(\frac{1}{t^{4}} x\right) d m(x)=1
$$

It increases very rapidly than the case of $\rho(t)=\frac{1}{t^{2}}(t>$ 1) , whenever a parameter $t>0$ is increasing, because 


$$
\mathrm{g}(2)=\left(\frac{8}{9}\right)^{\frac{n}{2}} \text { and } \mathrm{h}(2)=\left(\frac{128}{129}\right)^{\frac{n}{2}} \text { and } \mathrm{g}(2)<\mathrm{h}(2) \text {. }
$$

Remark. When I submitted the paper in [11], referee recommended to submit the paper to a more good journal, but I could not as I did not know the significant meaning of the change of scale for the Wiener integral! After then, I have had an effort to interpret the meaning of the scale more accurately.

Throughout this paper, I find a nice result for the homework about this query!

\section{CHANGE OF A SCALE FORMULA FOR THE WIENER INTEGRAL.}

In the year 1947, R. H. Cameron and W. T. Martin investigated the behavior of measure and measurability under change of scale in Wiener space.[Ref 5].

In 1987, R.H.Cameron and D.A.Storvick established the relationships between the Wiener Integral and the analytic Feynman integral [Ref.6] and proved a change of scale Formulas for the Wiener Integral.

From this motivation, we first define the scale factor for the Wiener integral.

In this section, we prove the relationships between the Wiener integral and the analytic Feynman integral[Ref.6]l and prove a change of scale Formulas for the Wiener Integral about the function in (3.1). Those relationships was first proved on some Banach algebra S in Ref 5 and in Ref 6.

Theorem 5.1. Let $\mathrm{F}: C_{0}[0, T] \rightarrow C$ be defined by (3.1) and (3.2). Then the analytic Wiener integral of $F(x)$ exists and is of the form:

$\int_{C_{0}[o, T[}^{a n w z} F(x) d m(x)$

$=\sum_{n=0}^{\infty} \int_{\Delta_{n}(T) \times R^{n}} \exp \left\{-\frac{1}{2 z} \sum_{j=1}^{n}\left(t_{j}-t_{j-1}\right) \sum_{k-j}^{n} v_{k}^{2}\right\} d \mu_{n}(\vec{t}, \vec{v})$

where $\mu_{n}$ is a countably additive Borel measure defined on $\Delta_{n}(T) \times R^{n}$ for each $\mathrm{n}=1,2, \ldots \mathrm{n}$.

Proof. In (3.6), we have that that for real $r>0$.,

$$
\begin{aligned}
& \int_{C_{0}[o, T]} F\left(r^{-\frac{1}{2} \cdot x}\right) d m(x) \\
& =\sum_{n=0}^{\infty} \int_{\Delta_{n}(T) \times R^{n}} \exp \left\{-\frac{1}{2 r} \sum_{j=1}^{n}\left(t_{j}-t_{j-1}\right) \sum_{k=j}^{n} v_{k}^{2}\right\} d \mu_{n}(\vec{t}, \vec{v})
\end{aligned}
$$

Now, we prove the existence of the analytic Wiener integral of $\mathrm{F}(\mathrm{x})$. For $\mathrm{r} \in C^{+}$, let

$$
\begin{gathered}
g_{r}=\prod_{j=1}^{n}\left(\frac{r}{2 \pi\left(t_{j}-t_{j-1}\right)}\right)^{\frac{1}{2}} \int_{R^{n}} \exp \left\{-\frac{r}{2} \sum_{j=1}^{n} \frac{\left[u_{j}-u_{j-1}\right]^{2}}{t_{j}-t_{j-1}}\right\} \\
\cdot\left[\sum_{n=0}^{\infty} \int_{\Delta_{n}(T) \times R^{n}} \exp \left\{i \sum_{j=1}^{n} u_{j} v_{j}\right\} d \mu_{n}(\vec{t}, \vec{v})\right] d \vec{u}
\end{gathered}
$$

which is a formula in the fourth equality in the equation (3.6). Let's show that $g_{r}$ is an analytic function of $r \in C^{+}$. Let $\Delta$ be any rectifiable simple closed curve lying in $C^{+}$and let $\alpha=\operatorname{Sup}\{r: r \in \Delta\}$ and $\beta=\{\operatorname{Re}(r): r \in \Delta\}$. Then for $r \in$ $C^{+}$,

$$
\begin{gathered}
\left|g_{r}\right| \leq \prod_{j=1}^{n}\left(\left|\frac{r}{2 \pi\left(t_{j}-t_{j-1}\right)}\right|\right)^{\frac{1}{2}} \\
\cdot \int_{R^{n}} \exp \left\{-\frac{\operatorname{Re}(r)}{2} \sum_{j=1}^{n} \frac{\left[u_{j}-u_{j-1}\right]^{2}}{t_{j}-t_{j-1}}\right\}\left(\sum_{n=0}^{\infty}\left\|\mu_{n}\right\|\right) d \vec{u} \\
=\prod_{j=1}^{n}\left(\left|\frac{r}{2 \pi\left(t_{j}-t_{j-1}\right)}\right|\right)^{\frac{1}{2}} \prod_{j=1}^{n}\left|\frac{2 \pi\left(t_{j}-t_{j-1}\right)^{\frac{1}{2}}}{\operatorname{Re}(r)}\right|\left(\sum_{n=0}^{\infty}\left\|\mu_{n}\right\|\right)<\infty
\end{gathered}
$$

As $g_{r}$ is a bounded function of $\mathrm{r} \in C^{+}, g_{r}$ is a continuous function of $r \in C^{+}$, by the Dominated Convergence Theorem. Moreover, by Fubini's Theorem and the Cauchy integral Theorem, we have that for $\mathrm{r} \in C^{+}, \int_{\Delta}\left|g_{r}\right| \mathrm{dr}=0$ for all rectifiable simple closed region $\Delta$ in $C^{+}$by Morera's Theorem. And therefore, the analytic Wiener integral $\int_{C_{0}[o, T \Gamma}^{a n w z} F(x) d m(x)$ exists for $\mathrm{z} \in C^{+}$and is given by (5.1).

Theorem5.2. Let F: $C_{0}[0, T] \rightarrow C$ be defined by (3.1) and (3.2). Then the analytic Feynman integral of $F(x)$ exists and is of the form : For real $q>0$,

$\int_{C_{0}[o, T[}^{a n f_{q}} F(x) d m(x)$

$=\sum_{n=0}^{\infty} \int_{\Delta_{n}(T) \times R^{n}} \exp \left\{-\frac{i}{2 q} \sum_{j=1}^{n}\left(t_{j}-t_{j-1}\right) \sum_{k-j}^{n} v_{k}^{2}\right\} d \mu_{n}(\vec{t}, \vec{v})$

where $\mu_{n}$ is a countably additive Borel measure defined on $\Delta_{n}(T) \times R^{n}$ for each $\mathrm{n}=1,2, \ldots \mathrm{n}$.

Proof. By the definition of the analytic Feynman integral and by the dominated convergence theorem, the analytic Feynman integral exists and is given by

$$
\int_{C_{0}[0, T]}^{\text {anfq }} F(x) d m(x)=\lim _{\mathrm{z} \rightarrow-\mathrm{iq}} \int_{C_{0}[0, T]}^{\text {anwz }} F(x) d m(x)
$$




$$
\begin{gathered}
=\lim _{\mathrm{z} \rightarrow-\mathrm{i} q} \sum_{n=0}^{\infty} \int_{\Delta_{n}(T) \times R^{n}} \exp \left\{-\frac{1}{2 r} \sum_{j=1}^{n}\left(t_{j}-t_{j-1}\right) \sum_{k=j}^{n} v_{k}^{2}\right\} d \mu_{n}(\vec{t}, \vec{v}) \\
=\sum_{n=0}^{\infty} \int_{\Delta_{n}(T) \times R^{n}} \exp \left\{-\frac{i}{2 q} \sum_{j=1}^{n}\left(t_{j}\right.\right. \\
\left.\left.-t_{j-1}\right) \sum_{k-j}^{n} v_{k}^{2}\right\} d \mu_{n}(\vec{t}, \vec{v})
\end{gathered}
$$

whenever $\mathrm{z} \rightarrow-\mathrm{iq}$ throughout $C^{+}$. Note that

$$
\left.\left|\int_{C_{0}[o, T[}^{a n f} f_{q} F(x) d m(x)\right| \leq \sum_{n=0}^{\infty}\left\|\mu_{n}\right\|<\infty \text { for real q }>0 .\right) .
$$

Now, we prove the following Lemma to prove the change of scale formula :

Lemma 5.3. Let $\mathrm{F}$ be defined by (3.1) and (3.2). Then for $\mathrm{z} \in C^{+}$,

$$
\exp \left\{\frac{1-z}{2} \sum_{j=1}^{n} \frac{\left[x\left(t_{j}\right)-x\left(t_{j-1}\right)\right]^{2}}{t_{j}-t_{j-1}}\right\} \cdot F(x)
$$

is a Wiener integrable function of $\mathrm{x} \in C_{0}[0, T]$. $C^{+}$,

Proof. By the Wiener integral formula, we have that for $z \in$

$$
\begin{aligned}
& \left|\int_{C_{0}[o, T[} \exp \left\{\frac{1-z}{2} \sum_{j=1}^{n} \frac{\left[x\left(t_{j}\right)-x\left(t_{j-1}\right)\right]^{2}}{t_{j}-t_{j-1}}\right\} \cdot F(x) d m(x)\right| \\
& \leq \mid \int_{C_{0}[o, T]} \exp \left\{-\frac{1-z}{2} \sum_{j=1}^{n} \frac{\left[x\left(t_{j}\right)-x\left(t_{j-1}\right)\right]^{2}}{t_{j}-t_{j-1}}\right\} \\
& \cdot\left(\sum_{n=0}^{\infty}\left\|\mu_{n}\right\|\right) d m(x) \\
& =\mid\left(\sum_{n=0}^{\infty}\left\|\mu_{n}\right\|\right) \cdot \prod_{j=1}^{n}\left(\left|\frac{1}{2 \pi\left(t_{j}-t_{j-1}\right)}\right|\right)^{\frac{1}{2}} \\
& \cdot \int_{R^{n}} \exp \left\{\frac{1-z}{2} \sum_{j=1}^{n} \frac{\left[u_{j}-u_{j-1}\right]^{2}}{t_{j}-t_{j-1}}\right\} \\
& \cdot \exp \left\{-\frac{1}{2} \sum_{j=1}^{n} \frac{\left[u_{j}-u_{j-1}\right]^{2}}{t_{j}-t_{j-1}}\right\} d \vec{u} \\
& =\left(\sum_{n=0}^{\infty}\left\|\mu_{n}\right\|\right) \cdot \prod_{j=1}^{n}\left(\left|\frac{1}{2 \pi\left(t_{j}-t_{j-1}\right)}\right|\right)^{\frac{1}{2}}
\end{aligned}
$$

$$
\begin{array}{r}
\int_{R^{n}} \exp \left\{-\frac{z}{2} \sum_{j=1}^{n} \frac{\left[x\left(t_{j}\right)-x\left(t_{j-1}\right)\right]^{2}}{t_{j}-t_{j-1}}\right\} d \vec{u} \\
=z^{-\frac{n}{2}} \cdot\left(\sum_{n=0}^{\infty}\left\|\mu_{n}\right\|\right)<\infty
\end{array}
$$

Therefore, we have the desired result.

Now, we prove the relationship between the analytic Wiener integral and the Wiener integral. And we show that the analytic Wiener integral can be expressed as the sequence of Wiener integrals.

Theorem 5.4. Let $\mathrm{F}$ be defined by (3.1) and (3.2). Then for $\mathrm{z} \in C^{+}$,

$$
\begin{gathered}
\int_{C_{0}[0, T]}^{a n w z} F(x) d m(x) \\
=z^{\frac{n}{2}} \cdot \int_{C_{0}[0, T]} \exp \left\{\frac{1-z}{2} \sum_{j=1}^{n} \frac{\left[x\left(t_{j}\right)-x\left(t_{j-1}\right)\right]^{2}}{t_{j}-t_{j-1}}\right\} \\
\cdot F(x) \mathrm{dm}(\mathrm{x})
\end{gathered}
$$

Proof. By the Wiener integration formula, we have that

$$
\begin{gathered}
\int_{C_{0}[0, T]} \exp \left\{\frac{1-z}{2} \sum_{j=1}^{n} \frac{\left[x\left(t_{j}\right)-x\left(t_{j-1}\right)\right]^{2}}{t_{j}-t_{j-1}}\right\} \cdot F(x) \mathrm{dm}(\mathrm{x}) \\
=\int_{C_{0}[0, T]} \exp \left\{\frac{1-z}{2} \sum_{j=1}^{n} \frac{\left[x\left(t_{j}\right)-x\left(t_{j-1}\right)\right]^{2}}{t_{j}-t_{j-1}}\right\}
\end{gathered}
$$

$\cdot \sum_{n=0}^{\infty} \int_{\Delta_{n}(T) \times R^{n}} \exp \left\{i \sum_{j=1}^{n} v_{j} x\left(t_{j}\right)\right\} d \mu_{n}(\vec{t}, \vec{v}) \operatorname{dm}(\mathrm{x})$

$$
=\prod_{j=1}^{n}\left(\frac{1}{2 \pi\left(t_{j}-t_{j-1}\right)}\right)^{\frac{1}{2}} \int_{R^{n}} \exp \left\{\frac{1-z}{2} \sum_{j=1}^{n} \frac{\left[u_{j}-u_{j-1}\right]^{2}}{t_{j}-t_{j-1}}\right\}
$$$$
\cdot \sum_{n=0}^{\infty} \int_{\Delta_{n}(T) \times R^{n}} \exp \left\{i \sum_{j=1}^{n} v_{j} u_{j}\right\} d \mu_{n}(\vec{t}, \vec{v})
$$$$
\cdot \exp \left\{-\frac{1}{2} \sum_{j=1}^{n} \frac{\left[u_{j}-u_{j-1}\right]^{2}}{t_{j}-t_{j-1}}\right\} d \vec{u}
$$

$$
\begin{aligned}
& =z^{-\frac{n}{2}} \\
& . \prod_{j=1}^{n}\left(\frac{z}{2 \pi\left(t_{j}-t_{j-1}\right)}\right)^{\frac{1}{2}} \int_{R^{n}} \exp \left\{-\frac{z}{2} \sum_{j=1}^{n} \frac{\left[u_{j}-u_{j-1}\right]^{2}}{t_{j}-t_{j-1}}\right\}
\end{aligned}
$$




$$
\begin{aligned}
& \cdot \sum_{n=0}^{\infty} \int_{\Delta_{n}(T) \times R^{n}} \exp \left\{i \sum_{j=1}^{n} v_{j} u_{j}\right\} d \mu_{n}(\vec{t}, \vec{v}) d \vec{u} \\
& =z^{-\frac{n}{2}} \cdot \prod_{j=1}^{n}\left(\frac{z}{2 \pi\left(t_{j}-t_{j-1}\right)}\right)^{\frac{1}{2}} \sum_{n=0}^{\infty} \int_{\Delta_{n}(T) \times R^{n}} \\
& \cdot \int_{R^{n}} \exp \left\{-\frac{z}{2} \sum_{j=1}^{n} \frac{\left[u_{j}-u_{j-1}\right]^{2}}{t_{j}-t_{j-1}}\right\} \\
& \left.\cdot \exp \left\{i \sum_{j=1}^{n} v_{j} u_{j}\right\} d \vec{u}\right] d \mu_{n}(\vec{t}, \vec{v}) \\
& =z^{-\frac{n}{2}} \\
& \cdot \sum_{n=0}^{\infty} \int_{\Delta_{n}(T) \times R^{n}} \exp \left\{-\frac{1}{2 z} \sum_{j=1}^{n}\left(t_{j-} t_{j-1)} \sum_{k=j}^{n} u_{k}^{2}\right\} d \mu_{n}(\vec{t}, \vec{v})\right. \\
& =z^{-\frac{n}{2}} \cdot \int_{C_{0}[0, T]}^{a n w z} F(x) d m(x)
\end{aligned}
$$

where the last equality follows from the Theorem 5.1.

Now, we prove the change of scale formula :

Theorem 5.5. Let $\mathrm{F}$ be defined by (3.1) and (3.2). Then for real $\rho>0$,

$$
\begin{gathered}
\int_{C_{0}[0, T]} F(\rho x) d m(x)=\rho^{-n} \\
\cdot \int_{C_{0}[0, T]} \exp \left\{\frac{\rho^{2}-1}{2 \rho^{2}} \sum_{j=1}^{n} \frac{\left[x\left(t_{j}\right)-x\left(t_{j-1}\right)\right]^{2}}{t_{j}-t_{j-1}}\right\} \cdot F(x) \mathrm{dm}(\mathrm{x}) .
\end{gathered}
$$

Proof. By (5.6), we have that for real $\mathrm{z}>0$,

$$
\begin{gathered}
\int_{C_{0}[0, T]} F\left(z^{-\frac{1}{2} x}\right) d m(x) \\
=z^{\frac{n}{2}} \cdot \int_{C_{0}[0, T]} \exp \left\{\frac{1-z}{2} \sum_{j=1}^{n} \frac{\left[x\left(t_{j}\right)-x\left(t_{j-1}\right)\right]^{2}}{t_{j}-t_{j-1}}\right\} \\
\cdot F(x) \operatorname{dm}(\mathrm{x})
\end{gathered}
$$

If we let $\mathrm{z}=\rho^{-2}$ in (5.8), we can have the desired result.
Finally, we prove the relationship between the analytic Feynman integral and the analytic Wiener integral. That is, we show that the analytic Feynman integral can be successfully expressed as the limit of the sequence of analytic Wiener integrals.

Theorem 5.6. Let F be defined by (3.1) and (3.2). Then

$$
\begin{aligned}
& \int_{c_{0}[0, T]}^{\operatorname{anf} q} F(x) d m(x) \\
&=\lim _{z_{k} \rightarrow-i q} z_{k}^{\frac{n}{2}} \cdot \int_{c_{0}[0, T]}^{a n w z_{k}} \exp \left\{\frac{1-z_{k}}{2} \sum_{j=1}^{n} \frac{\left[x\left(t_{j}\right)-x\left(t_{j-1}\right)\right]^{2}}{t_{j}-t_{j-1}}\right\} \\
& \cdot F(x) \mathrm{dm}(\mathrm{x})
\end{aligned}
$$

Proof. By the definition of the analytic Feynman integral and exploiting the proof of the Theorem 5.4, we can deduce that

$$
\begin{gathered}
\int_{C_{0}[0, T]}^{\text {anf } q} F(x) d m(x) \\
=\lim _{\mathrm{z} z_{k} \rightarrow-\mathrm{iq}} \int_{C_{0}[0, T]}^{a n w z_{k}} F(x) d m(x) \\
=\lim _{z_{k} \rightarrow-i q} z_{k}^{\frac{n}{2}} \cdot \int_{c_{0}[0, T]}^{a n w z_{k}} \exp \left\{\frac{1-z_{k}}{2} \sum_{j=1}^{n} \frac{\left[x\left(t_{j}\right)-x\left(t_{j-1}\right)\right]^{2}}{t_{j}-t_{j-1}}\right\} \\
\cdot F(x) \mathrm{dm}(\mathrm{x})
\end{gathered}
$$

Finally, we introduce the Motivation and the Application of the Wiener integral :

\section{Remark.}

(1) Motivation: The solution of the heat equation $\frac{\partial U}{\partial t}=$ $-H U, U(0, \cdot)=\varphi(\cdot)$ is

$$
U(t, \varepsilon)=\left(e^{-t H} \varphi\right)(\varepsilon)=E\left[e^{-\int_{0}^{t} V(x(s)+\varepsilon) d s} \cdot \varphi(x(t)+\varepsilon)\right],
$$

where $\varphi \in L_{2}\left[R^{d}\right]$ and $\varepsilon \in R^{d}$ and $\mathrm{x}(\cdot)$ is a $R^{d}$ - valued continuous function defined on $[0, \mathrm{t}]$ such that $\mathrm{x}(0)=0$ and $\mathrm{E}$ denotes the expectation with respect to the Wiener path starting at time $t=0$ and

$H=-\Delta+\mathrm{V}$ is the energy operator(or, Hamiltonian) and $\Delta$ is a Laplacian and $\mathrm{V}: R^{d} \rightarrow R$ is a potential. This formula (4.12) is called the Feynman-Kac formula.

(2). Application of the Feynman-Kac formula(in various settings) have been given in the area : diffusion equation, the spectral theory of 
the schrodinger operator, quantum mechanics, statistical physics.(For more details, see the paper [8] and the book [9]

\section{ACKNOWLEDGMENT}

THANKS 1. I am very gratitude to professors : R.H. Cameron and M.T.Martin and D.A. Storvick in [2] [7] who give me a very nice research area about the structure of the change of scale and the change of scale formula and about the every harmonious relationships among the Wiener integral and the analytic Wiener integral and the analytic Feynman integral.

Those papers give me an excellent motivation about the new concept of the scale factor for the Wiener integral along the path of a parameter $t>0$ !!

THANKS 2. I am very gratitude to professors, G.W. Johnson and M.L.Lapidus to research about the Feynman integral theory from their book in [9].

THANKS 3. I am very gratitude to the National Research Foundation of the Republic of Korea supporting the research fund for my work.

Research fund of this paper is supported by NRF2017R1A6A3A11030607.

\section{REFERENCES}

[1] M.D.Brue, A functional transform for Feynman integrals similar to Fourier transform, Thesis (1972). University of Minnesota.

[2] R. H. Cameron, The translation pathology of Wiener space. Duke Math J. 21 (1954) 623-628.

[3] R. H. Cameron and W. T. Martin, On transformations of Wiener integrals under translations. Ann. of Math. 45, (1944) 386-396.

[4] R. H. Cameron and W. T. Martin, Transformations for Wiener integrals under a general class of linear transformations. Trans. Amer. Math. Soc. vol. 58, no.2, (1945 ) 184-219.

[5] R. H. Cameron and W. T. Martin, The behavior of measure and measurability under change of scale in Wiener space. Bull. Amer. Math. Soc. 53, (1947). 130-137.

[6] R.H.Cameron and D.A.Storvick, Relationships between the Wiener Integral and the Analytic Feynman Integral. Supplemento ai Rendiconti del Circolo Matematico di Palermo, Serie II-numero. 17. (1987), 117133.

[7] R.H.Cameron and D.A.Storvick, Change of scale Formulas for Wiener Integral. Supplemento ai Rendiconti del Circolo Matematico di Palermo, Serie II-numero. 17. (1987), 105-115.

[8] M.D. Gaysinsky and M.S. Goldstein, Self-Adjointness of Schrl"\{o\}dinger Operator and Wiener Integrals. Integral Equation Operator Theory. 15, (1992 ), 973-990.

[9] G.W. Johnson and M.L. Lapidus, The Feynman integral and Feynman's Operational Calculus, Oxford Science Publications. (2000).

[10] G.W.Johnson and D.L.Skoug, Scale-invariant Measurability in Wiener Space, Pacific J. Math. 283, (1979), 157-176.

[11] G.Kallianpur and C.Bromley, Generalized Feynman Integrals Using Analytic Continuation in Several Complex Variables, In Stochastic Analysis and Applications (ed. M.H.Pinsky), Dekker, New York. (1984), 217-267.

[12] T..Huffmann, C.Park and D.Skoug, Convolutions and Fourier Feynman transforms. Rocky Mountain Jour. of Math. .27, No 3, (1997), 827-841.

[13] Y. S. Kim, A change of scale formula for Wiener Integrals of cylinder functions on the abstract Wiener space. Internat. J. of Math. and Math.Sci. 21, No 1, (1998), 73-78.

[14] Y. S. Kim, A change of scale formula for Wiener Integrals of cylinder functions on abstract Wiener space II. International Journal of Mathematics and Mathematical Sciences. 25, No 4, (2001), 231-237.
[15] Y. S. Kim, The behavior of the first variation under the Fourier Feynman transform on abstract Wiener spaces. Journal of Fourier Analysis and Application. 12, No 3,\} (2006), 233-242.

[16] Y .S. Kim, Fourier Feynman Transform and analytic Feynman integrals and convolutions of a Fourier transform of a measure on Wiener spaces. Houston Journal of Mathematics. 36, No 1. (2010). 1139-1158.

[17] Y. S. Kim, Behavior of the first variation under a Fourier-Feynman transform for cylinder functions on Wiener spaces II.. Integral Transforms and Special Functions. 21. No 1, (2010), 13-23.

[18] Y. S. Kim, Behavior of the first variation of Fourier transform of a measure on the Fourier Feynman transform and convolution. Numerical Functional Analysis and Optimization. 37, No 6. (2016). 699-718. 